\title{
Substituição parcial do pericárdio de cães por membrana de látex natural
}

\author{
Soraya Lopes SADER*, Joaquim COUTINHO NETTO*, José BARBIERI NETO*, Sebastião Assis \\ MAZZETTO*, Paulo ALVES JR. ${ }^{*}$, José Carlos VANNI*, Albert Amim SADER*
}

RBCCV 44205-520

Sader S L, Coutinho Netto J, Barbieri Neto J, Mazzetto S A, Alves Jr. P, Vanni J C, Sader A A. Substituição parcial do pericárdio de cães por membrana de látex natural. Rev Bras Cir Cardiovasc 2000; 15(4): 338-44.

RESUMO: Fundamento: São numerosas as vantagens de se fechar a cavidade pericárdica após as operações cardíacas e pneumonectomias intrapericárdicas.

Objetivo: Estudar o comportamento da membrana de látex natural como substituto parcial do pericárdio.

Material e Métodos: Em 16 cães, divididos em 3 grupos, ressecou-se um retalho elíptico da porção ântero-lateral esquerda do pericárdio $(7 \mathrm{~cm} \times 5 \mathrm{~cm})$ : Grupo $A(n=4)-0$ retalho removido foi reimplantado imediatamente; Grupo $B(n=8)$ - o retalho foi substituído por outro, de látex natural, com área equivalente e espessura de $0,3 \mathrm{~mm}$; Grupo $\mathrm{C}(\mathrm{n}=4)$ - retalho de látex de $0,7 \mathrm{~mm}$ de espessura. Em todos os animais, fixouse o retalho com sutura contínua de fio de polipropileno 5-0 ou 6-0. No último grupo, foram dados 4 pontos adicionais, em U, ancorados em barras de dacron. Realizaram-se eletrocardiogramas (ECG) e leucogramas no pré e no pós-operatório, bem como estudo macro e microscópio post-mortem.

Resultados: Grupo A - auto-enxerto íntegro, macro e microscopicamente, fortemente aderido ao pulmão e frouxamente aderido ao epicárdio; Grupo B - deiscência parcial da sutura em 1 e total em 2 animais. A membrana de látex não aderiu nem ao pulmão nem ao epicárdio. Em $3(37,5 \%)$ animais houve regeneração total e distinta do pericárdio subjacente ao látex, microscopicamente idêntico ao pericárdio nativo. Grupo $\mathrm{C}$-suturas íntegras. Em 3 (75\%) animais observou-se regeneração pericárdica total e distinta. Nos demais animais dos Grupos B e C, e epicárdio subjacente ao látex apresentava-se ligeiramente espessado, permitindo visibilizar os vasos coronários com facilidade. Microscopicamente, identificaram-se algumas áreas com denso infiltrado linfoplasmocitário, proliferação fibroblástica e vascular; em outras, os fibroblastos circunscreviam fendas com revestimento mesotelial, indicativas de regeneração irregular do pericárdio. Não se observou infecção local nem alteração do leucograma e ao ECG, apenas inversão da onda T, em todos os grupos.

Conclusão: A membrana de látex natural mostrou-se adequada para a substituição parcial do pericárdio de cães, em observação de até 345 dias, propiciando a regeneração do pericárdio nativo.

DESCRITORES: Substitutos do pericárdio. Próteses e implantes. Pericárdio. Retalhos cirúrgicos. Procedimentos cirúrgicos cardíacos, métodos. Procedimentos cirúrgicos cardíacos, efeitos adversos.

\section{INTRODUÇÃO}

O pericárdio, cuja principal função é conter o coração e permitir que deslize com mínimo atrito, não é indispensável ao seu funcionamento. Contudo, sua falta, ainda que parcial, pode ser a causa de importantes problemas, que os cirurgiões torácicos e cardiovasculares devem evitar ou resolver.

Trabalho realizado na Faculdade de Medicina de Ribeirão Preto, da Universidade de São Paulo. Ribeirão Preto, SP, Brasil. Apresentado no $27^{\circ}$ Congresso Nacional de Cirurgia Cardíaca. Rio de Janeiro, RJ, 23 a 25 de março de 2000.

* Da Faculdade de Medicina de Ribeirão Preto.

Endereço para correspondência: Soraya Lopes Sader. Rua José Lesal, 1015. Ribeirão Preto, SP, Brasil. CEP 14025-260. Tel. (16) 623-1360. Fax. (16) 610-6609. e-mail: slsader@hotmail.com. 
Sader S L, Coutinho Netto J, Barbieri Neto J, Mazzetto S A, Alves Jr. P, Vanni J C, Sader A A. - Substituição parcial do pericárdio de cães por membrana de látex natural. Rev Bras Cir Cardiovasc 2000; 15(4): 338-44.

Nas pneumectomias radicais com ressecção parcial do pericárdio não reparada, pode ocorrer hérnia e estrangulamento do coração. Este quadro, extremamente grave, freqüentemente culmina com a morte dos pacientes, mesmo quando reoperados precocemente (1-3).

Nas operações cardíacas, geralmente realizadas por esternotomia, a face anterior do coração e grandes vasos pode ficar sem a proteção do pericárdio nas seguintes situações: 1) quando o pericárdio é usado para reparar os defeitos cardíacos; 2) nas reoperações múltiplas; 3) nas operações em que se fazem condutos extracardíacos e nas de revascularização do miocárdio, quando se deixa o pericárdio aberto para que não se comprimam os condutos e os enxertos vasculares; 4) quando, no final da operação, ocorre aumento de volume do coração e o fechamento constrito do pericárdio implica em prejuízo à função diastólica do coração (4). Embora nessas circunstâncias os órgãos e estruturas pericárdicos mantenham o coração devidamente alojado, outros problemas podem surgir no pós-operatório imediato e tardio. O pericárdio amplamente aberto aumenta os riscos de tamponamento cardíaco; não permite discriminar o sangramento intra e extrapericárdico; aumenta a incidência da síndrome pós-pericardiotomia (5) e propicia a formação de aderências entre o esterno e o ventrículo direito, reduzindo sua fração de ejeção (6). Essa fixação do esterno aos órgãos subjacentes implica em maiores riscos nas reoperações por esternotomia, cada vez mais freqüentes $(7,8)$. Levantamento multicêntrico feito por DOBEL \& JAIN (9) evidenciou que, de 144 casos de hemorragia catastrófica ocorrida durante a reesternotomia, $88 \%$ não tiveram o pericárdio fechado na primeira operação.

Esses são os motivos pelos quais tem-se buscado maneiras de se fechar a cavidade pericárdica com técnicas diversas de pericardioplastia (10-12) e com substitutos do pericárdio, biológicos e sintéticos. Os primeiros podem ser autólogos $(7,13,14)$ ou heterólogos (15-21), os últimos, inabsorvíveis (22-27) e absorvíveis (27-30).

A diversidade dos materiais usados aponta para o fato de que nenhum preencheu ainda os requisitos necessários a um substituto ideal do pericárdio ${ }^{(22)}$.

O látex é considerado um material menos nobre do que os sintéticos usados anteriormente. Contudo, o seu bom desempenho em próteses parciais do esôfago (31) despertou o interesse de experimentálo como substituto do pericárdio.

\section{MATERIAL E MÉTODOS}

Dezesseis cães mestiços, de ambos os sexos, com peso médio de $11,3 \mathrm{Kg}$, foram anestesiados com thiopental e halotano. Com a traquéia intubada e conectada a um respirador Takaoka Mod. 600, precedeu-se à toracotomia esquerda no $5^{\circ}$ espaço intercostal. Ressecou-se um retalho do pericárdio, tão amplo quanto possível (média $7 \times 5 \mathrm{~cm}$ ), mobilizando-se, por vezes, o nervo frênico esquerdo.

Distribuíram-se os animais em 3 grupos: Grupo A $(n=4)$, no qual o retalho removido foi reimplantado imediatamente; GRUPO B $(n=8)$, em que o retalho removido foi substituído por outro, de látex natural, com área equivalente e espessura aproximada de $0,3 \mathrm{~mm}$; Grupo $\mathbf{C}(\mathrm{n}=4)$, no qual o retalho de látex tinha aproximadamente $0,7 \mathrm{~mm}$ de espessura. Em todos os animais fixou-se o retalho com sutura contínua, em chuleio, usando-se fio de polipropileno 5-0 ou 6-0, deixando-se uma abertura de $2 \mathrm{~cm}$ para drenagem do líqüido pericárdico. No último grupo, foram dados 4 pontos adicionais, em $U$, ancorados em barras de "dacron".

Realizaram-se traçados eletrocardiográficos antes e depois do ato operatório e antes do sacrifício dos animais. Amostras do sangue venoso para o leucograma foram obtidas antes da operação e 5 dias depois.

Os animais foram observados durante períodos variáveis. No grupo $A$, um animal foi sacrificado no $10^{\circ}$ dia e os outros foram mantidos vivos durante 303, 324 e 330 dias. No grupo B, o período de observação variou entre 25 e 345 dias (média 215 dias). No grupo $\mathrm{C}$, mantiveram-se os animais vivos durante 10, 12, 105 e 150 dias.

Findo o período de observação, os animais foram anestesiados e reoperados por esternotomia mediana. Procurou-se observar: 1) aderências no pericárdio reimplantado e da membrana de látex ao pulmão e ao epicárdio; 2) as condições do pericárdio remanescente e do pericárdio reimplantado; 3) as condições da membrana de látex e das estruturas subjacentes. Concluídas as observações, os animais foram sacrificados e o coração retirado juntamente com o pericárdio, para o exame histológico pertinente.

\section{Exame Histológico}

Fragmentos do pericárdio nativo, do pericárdio reimplantado, da membrana de látex e das estruturas a ela subjacentes, foram estudados por microscopia óptica após fixação em formol neutro a $10 \%$, e coloração pela hematoxilina de Harris e eosina. 


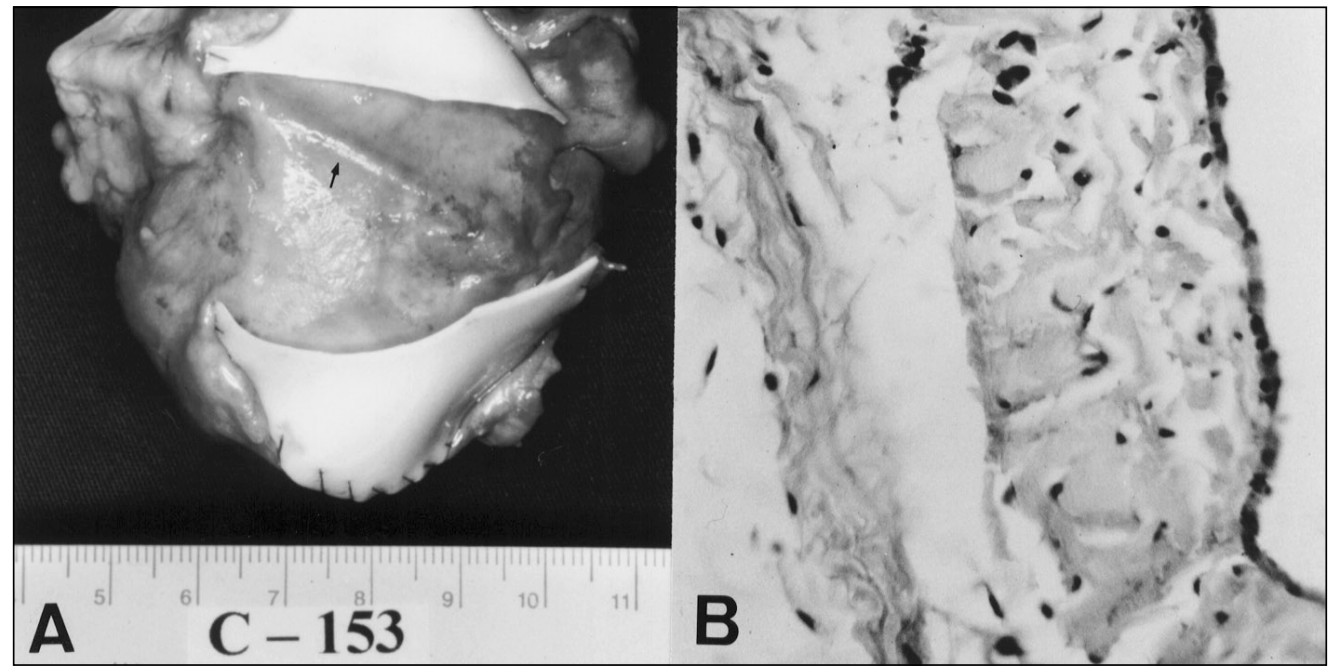

Fig. 1-A - Fotografia da peça de um animal do grupo C, retirada 105 dias após a operação. Vê-se o pericárdio regenerado sob a membrana de látex natural, parcialmente removida. Um cateter insinuado entre o epicárdio e o neoepicárdio (seta) comprova a separação das duas membranas. O número do animal é o do biotério. B - Fotomicrografia do pericárdio neoformado, observando-se, à direita, o revestimento mesotelial e, no restante, o tecido de sustentação formado por conjuntivo denso, colagenizado. $\mathrm{H}-\mathrm{E} \times 340$.

\section{Membrana de Látex Natural}

Confeccionada artesanalmente no Departamento de Bioquímica da Faculdade de Medicina de Ribeirão Preto - USP, a partir da seiva da Havea brasiliensis obtida na região de São José do Rio Preto. A seiva era colhida com amônia para, alcalinizando-a $(\mathrm{pH}=9-10)$, evitar coagulação à temperatura ambiente. Levada ao laboratório em recipiente de plástico hermeticamente fechado, adicionava-se a ela, lentamente e sob agitação branda, uma suspensão saturada de enxofre em água, na proporção de $20 \mathrm{~g} / \mathrm{l}$. Essa emulsão era, então, colocada em placas de Petri, em quantidades suficientes para a confecção das membranas com a espessura desejada $(0,3 \mathrm{~mm}$ ou $0,7 \mathrm{~mm})$. Para a polimerização do látex, deixavam-se placas de Petri em estufa ventilada, a $55^{\circ} \mathrm{C}$, durante 60 minutos, após os quais as membranas eram resfriadas à temperatura ambiente, empacotadas individualmente e esterilizadas com óxido de etileno.

Com os recursos disponíveis, não se conseguiram membranas com espessura uniforme, e suas propriedades elásticas foram avaliadas, após a remoção, pela simples manobra de distensão.

\section{RESULTADOS}

Grupo A - Em todos os animais, o auto-enxerto do pericárdio encontrava-se firmemente aderido ao pulmão esquerdo e frouxamente aderido ao epicárdio subjacente, particularmente nas linhas de sutura.
Tanto macro quanto microscopicamente, o retalho não se distinguia do pericárdio normal remanescente.

Grupo B - A membrana de látex não aderiu nem ao pulmão nem ao epicárdio subjacente, exceto nas linhas de sutura. Observou-se deiscência parcial da sutura em 2 cães e total em 1, no qual a membrana encontrava-se inteiramente destacada, próxima do local implantado. Neste, com 345 dias de evolução, e em outros 2 animais com 25 dias e 290 dias de evolução $(37,5 \%)$, houve regeneração completa do pericárdio subjacente à membrana de látex, cujas características macro e microscópicas se assemelham às do pericárdio nativo (Figuras 1-A e B). O neopericárdio se distinguia facilmente do epicárdio e as mínimas e frouxas aderências, quando presentes, encontravam-se próximas à linha de sutura. $O$ epicárdio subjacente apresentava transparência normal.

Grupo C - Nos cães deste grupo, as suturas estavam íntegras e não havia aderências da membrana de látex ao pulmão e, na outra face, apenas as linhas de sutura encontravam-se parcialmente aderidas ao epicárdio. Em 3, de 4 (75\%) animais, houve regeneração pericárdica. No cão com 105 dias de evolução, a regeneração foi completa, como nos 3 animais do grupo B; naqueles, mortos 10 e 12 dias após o implante, identificou-se uma delicada membrana transparente, situada internamente ao retalho de látex e aderida, em continuidade, ao pericárdio nativo (Figura 2-A). Os cortes histológicos dessa membrana neoformada, evidenciaram proliferação fibroblástica e vascular, com intensidade decrescente em sentido centrípeto. Mesma distribuição tinha o revestimento mesotelial, às vezes presente nas 2 
Sader S L, Coutinho Netto J, Barbieri Neto J, Mazzetto S A, Alves Jr. P, Vanni J C, Sader A A. - Substituição parcial do pericárdio de cães por membrana de látex natural. Rev Bras Cir Cardiovasc 2000; 15(4): 338-44.

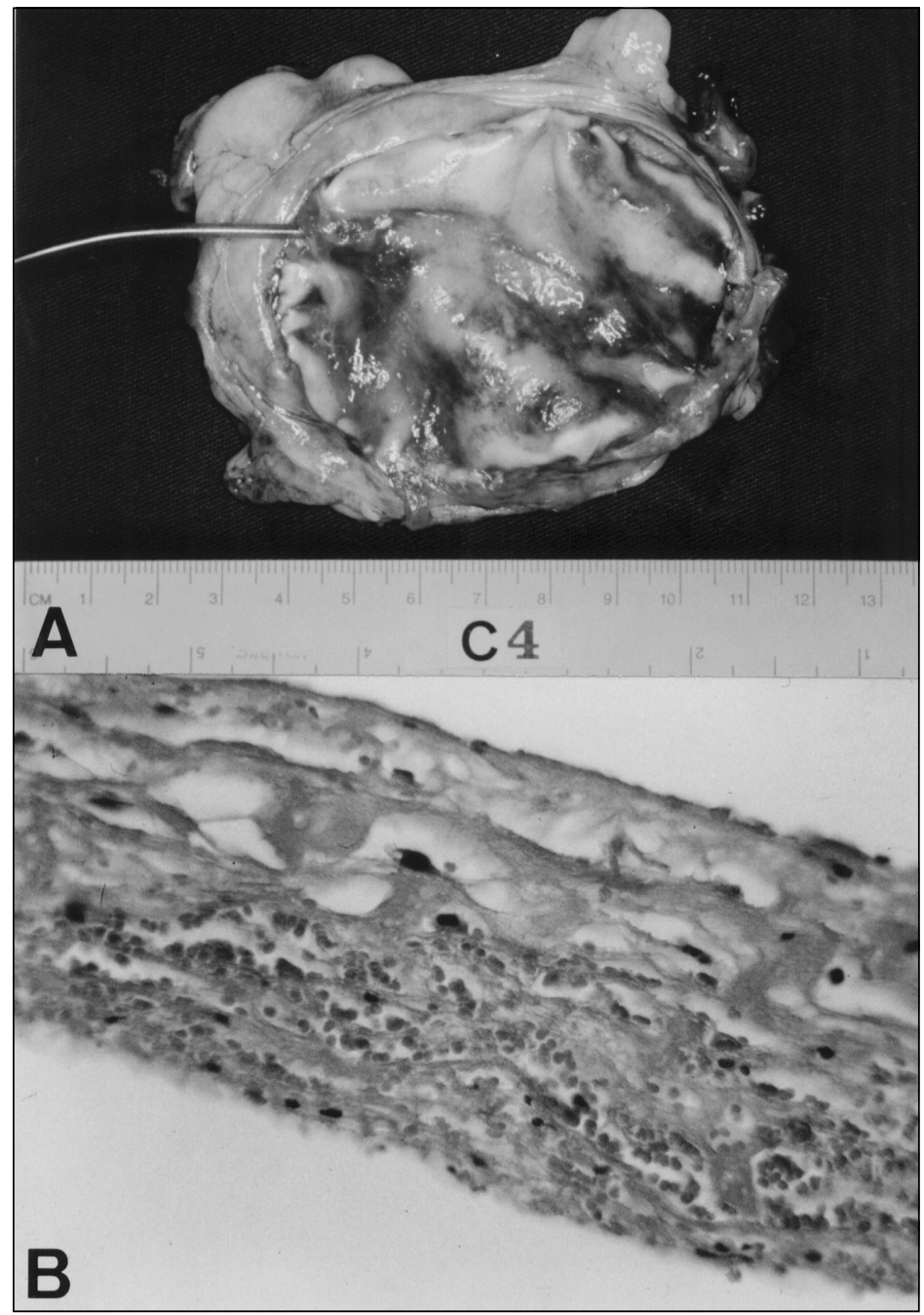

Fig. 2-A - Membrana de látex natural, neopericárdio em formação e parte do pericárdio nativo, vistos pela face interna, removidos de um animal do grupo C, 12 dias após a operação. O cateter foi introduzido entre a membrana de látex e o pericárdio neoformado, aderidos na linha de sutura, apenas. B - Fotomicrografia do neopericárdio em fase precoce de formação, vendo-se o material colagenizado e fibroblastos em meio à proliferação vascular. H-E x 340 .

faces (Figura 2-B). Nos demais cães dos grupos B e $\mathrm{C}$, o epicárdio subjacente à membrana de látex apresentava-se ligeiramente espessado, permitindo, contudo, visibilizar facilmente os vasos coronários (Figura 3-A). Microscopicamente, identificaram-se algumas áreas com denso infiltrado linfo-plasmocitário e proliferação fibroblática e vascular revestida por fibrina. Em outros locais, os fibroblastos circunscreviam fendas e recessos revestidos por células mesoteliais bem definidas (Figura 3-B).

Não se observou nem infecção local, nem alteração do leucograma em qualquer animal e, no eletrocardiograma constatou-se, apenas, inversão da onda $\mathrm{T}$ em todos os grupos.
A membrana de látex natural conservou suas propriedades elásticas mesmo após o período de observação mais longo, aumentando-se o seu comprimento em até 6 vezes, sem se romper.

\section{COMENTÁRIOS}

Já não se discute o fato de que a cavidade pericárdica deva ser fechada após as operações torácicas, particularmente as cardíacas. Preferencialmente, deve-se fazer a sutura direta das bordas do pericárdio quando isso não provocar compressão do conteúdo da cavidade pericárdica. A segun- 


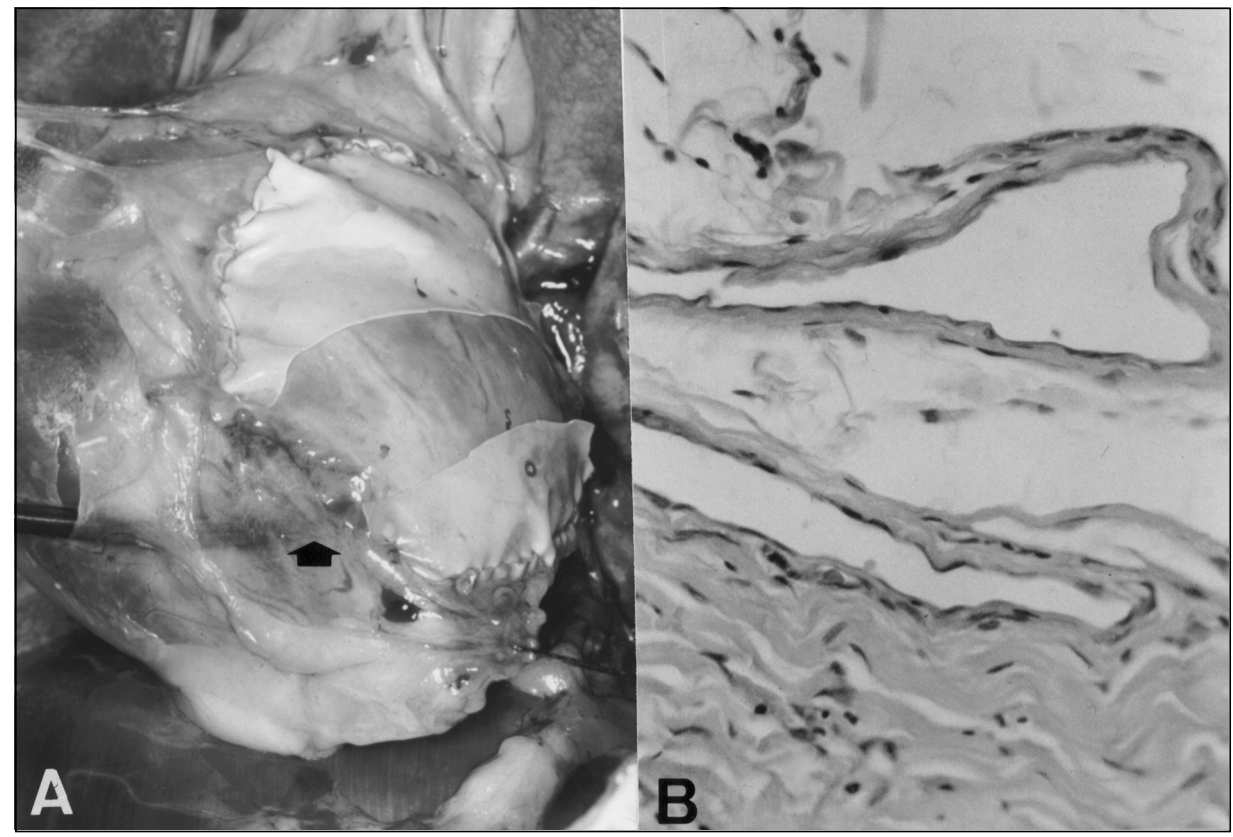

Fig. 3-A - Fotografia tomada após a reabertura do tórax de um animal do grupo B, operado 290 dias antes. O cateter, introduzido na cavidade pericárdica em direção à linha de sutura, não a ultrapassa (seta). Os vasos coronários podem ser vistos na superfície do coração, na área correspondente à membrana de látex natural, parcialmente removida. B - Fotomicrografia do tecido que reveste o coração sob a membrana de látex natural. Vê-se, em meio ao tecido conjuntivo, uma fenda e um recesso revestidos por células mesoteliais. H-E x 340 .

da opção, quando possível, recai sobre as pericardiopleuroplastias ${ }^{(10-12)}$, mais adequadas nas operações cardíacas, nas quais a presença de ambos os pulmões e o pericárdo remanescente garantem a posição ortotópica do coração. O mesmo não se pode dizer no caso das pneumectomias radicais intrapericárdiais, nas quais um retalho pleural delgado pode ser insuficiente para evitar hérnia e estrangulamento cardíacos ${ }^{(2)}$. Por último, os substitutos do pericárdio, orgânicos e sintéticos. Destes, os reabsorvíveis parecem oferecer os melhores resultados, experimentalmente, constituindo-se numa ponte para a completa regeneração do pericárdio $(28,29)$. Contudo, a experiência clínica é muito limitada e restrita a um período de observação insuficiente para conclusões definitivas ${ }^{(27}$, 30). Nesses trabalhos, não há referências a reoperações. Com relação aos inabsorvíveis, a membrana cirúrgica de politetrafluoretileno (PTFE) é a que tem apresentado comportamento mais satisfatório $(20,26,27,32)$. Contudo, MEUS et al.(25) observaram, no cão, que a membrana de PTFE provoca intensa reação epicárdica que impede o reconhecimento dos vasos coronários subjacentes. Para eles, os melhores resultados foram obtidos com o pericárdio bovino, cujas reações adversas foram apontadas por GALO et al. ${ }^{(4)}$, SKINNER et al. (17) e ENG et al. (33), quando usado no ser humano.
O látex é um material amplamente usado na prática médico-hospitalar, na forma de luvas, sondas, êmbolos de seringas, etc., em contato temporário com os tecidos humanos. Em sua forma pura, não tem sido empregado em implantes permanentes. O látex natural usado por MRUÉ (31) como prótese de um segmento do esôfago cervical de cães não foi incorporado aos tecidos do hospedeiro, funcionando apenas como ponte para a regeneração dos tecidos. Foi eliminado entre 12 e 175, juntamente com o bolo fecal. Essa extraordinária propriedade indutora da regeneração tecidual motivou a presente pesquisa.

Apesar de a sensibilidade ao látex ou aos seus aditivos ser um fenômeno freqüente $(34,35)$, não se observou em nenhum animal qualquer reação adversa. Não ocorreu nem infecção local nem alterações sensíveis do leucograma e as alterações eletrocardiográficas foram semelhantes nos 3 grupos.

A membrana não aderiu nem ao pulmão nem ao epicárdio, salvo nas linhas de sutura, onde ela se incorporou ao pericárdio nativo. Em 6 (50\%) de 12 animais dos grupos $\mathrm{B}$ e $\mathrm{C}$ houve completa regeneração do pericárdio subjacente à membrana de látex, bem individualizada macroscopicamente, que em 2 animais pôde ser identificada 10 e 12 dias após a operação. Nos cães restantes, em meio ao discreto espessamento epicárdico, observaram-se fendas e 
Sader S L, Coutinho Netto J, Barbieri Neto J, Mazzetto S A, Alves Jr. P, Vanni J C, Sader A A. - Substituição parcial do pericárdio de cães por membrana de látex natural. Rev Bras Cir Cardiovasc 2000; 15(4): 338-44.

recessos revestidos por células idênticas às mesoteliais, configurando um quadro de regeneração irregular do pericárdio. Pode-se especular se, com o tempo, essas formações se confluirão para formar uma membrana contínua ou se, como hipótese mais provável, elas resultaram de aderências do neopericárdio ao epicárdio.

Essa propriedade indutora da regeneração tecidual, cujos resultados são altamente vantajosos em casos de reoperação, não foi observada com nenhum outro material inabsorvível, nos trabalhos da literatura consultada.

\section{CONCLUSÃO}

\begin{abstract}
A membrana de látex mostrou-se satisfatória para a substituição parcial do pericárdio de cães, em observação de até 345 dias, propiciando a regeneração do pericárdio nativo.
\end{abstract}

\section{RBCCV 44205-520}

Sader S L, Coutinho Netto J, Barbieri Neto J, Mazzetto S A, Alves Jr. P, Vanni J C, Sader A A Partial replacement of dog pericardium with a natural latex membrane. Rev Bras Cir Cardiovasc 2000; 15(4): 338-44.

ABSTRACT: Background: There are numerous advantages in closing the pericardial cavity after heart surgery and intrapericardial pneumonectomies.

Objective: To study the behavior of a natural latex membrane as a partial pericardium substitute.

Material and Methods: Sixteen dogs divided into 3 groups were submitted to resection of an elliptical flap of the left anterolateral portion of the pericardium $(7 \times 5 \mathrm{~cm})$ : Group $A(n=4)$-the removed flap was immediately reimplanted; Group B $(n=8)$ - the flap was replaced with a $0.3 \mathrm{~mm}$ thick natural latex membrane of equivalent area; Group C $(n=4)$ - the latex flap was $0.7 \mathrm{~mm}$ thick. In all animals the flap was fixed with continuous 5-0 or 6-0 polypropylene sutures. In group C, 4 additional U-shaped stitches anchored with dacron pledgets were applied. All animals were submitted to ECG and to white cell counts during the preoperative period as well as to macro- and microscopic post mortem study.

Results: Group A - the self-graft was macro and microscopically intact, strongly adhering to the lung and loosely adhering to the epicardium; Group B - total suture dehiscence occurred in 1 animal and partial dehiscence in 2. The latex membrane did not adhere to the lung or to the epicardium. In 3 animals $(37.5 \%)$ there was full regeneration of the pericardium underlying the natural latex, microscopically identical to the native pericardium. Group C - the sutures were intact and total pericardium regeneration was observed in 3 animals (75\%). In the remaining animals of Groups $\mathrm{B}$ and $\mathrm{C}$, the epicardium underlying the latex was slightly thickened, permitting easy visualization of the coronary vessels. Some areas with dense lymphocytes and plasma cells infiltrates, fibroblast and vascular proliferation were microscopically identified in its thickness; in some other areas the fibroblasts surrounded slits with mesothelial lining indicating irregular pericardium regeneration. No local infection or changes in white cell counts were observed and the ECG only showed T wave inversion in all groups.

Conclusion: The natural latex membrane proved to be adequate for partial pericardium replacement in dogs kept under observation for up to 345 days, favoring regeneration of the native pericardium.

DESCRIPTORS: Pericardial substitutes. Prostheses and implants. Pericardium. Surgical, flaps. Cardiac surgical procedures, methods. Cardiac surgical procedures, adverse effects.

AGRADECIMENTO: Os autores agradecem à Sra. Rosângela Orlandin Lopes, responsável pela técnica histológica deste trabalho.

\section{REFERÊNCIAS BIBLIOGRÁFICAS}

1 Sharma V N, Bates M, Hurt R L - Herniation of the heart after intrapericardial pneumonectomy for bronchial carcinoma. Thorax 1959; 14: 36-8.

2 Yacoub M H, William W G, Ahmad A - Strangulation of the heart following intrapericardial pneumonectomy. Thorax 1968; 23: 261-5.
3 Deiraniya A K - Cardiac herniation following intrapericardial pneumonectomy. Thorax 1974; 29: 545-52.

4 Gallo I, Artinano E, Duran C G - Late clinical results with the use of heterologous pericardium for closure of the pericardial cavity. J Thorac Cardiovasc Surg 1985; 89: 709-12.

5 Cunningham Jr. J N, Spencer F C, Zeff R, Williams $C D$, Cukingnan $R$, Mullin $M$ - Influence of primary closure of the pericardium after openheart surgery on the frequency of tamponade, postcardiotomy syndrome, and pulmonary complications. J Thorac Cardiovasc Surg 1975; 70: 119-25. 
Sader S L, Coutinho Netto J, Barbieri Neto J, Mazzetto S A, Alves Jr. P, Vanni J C, Sader A A. - Substituição parcial do pericárdio de cães por membrana de látex natural. Rev Bras Cir Cardiovasc 2000; 15(4): 338-44.

6 Bailey L L, Ze-jian L, Schulz E, Roost H, Yahiku P - A cause of right ventricular dysfunction after cardiac operations. J Thorac Cardiovasc Surg 1984; 87: 539-42.

7 Culliford A T \& Spencer F C - Guidelines for safely opening a previous sternotomy incision. $J$ Thorac Cardiovasc Surg 1979; 78: 633-8

8 Londe $\mathrm{S} \&$ Sugg $\mathrm{W} \mathrm{L}$ - The challenge of reoperation in cardiac surgery. Ann Thorac Surg 1974; 17: 157-62.

9 Dobell A R \& Jain A K - Catastrophic hemorrhage during redo sternotomy. Ann Thorac Surg 1984; 37: 273-8.

10 Milgalter E, Uretzky G, Siberman S et al. - Pericardial meshing: an effective method for prevention of pericardial adhesions and epicardial reaction after cardiac operations. J Thorac Cardiovasc Surg 1985; 90: 281-6.

11 DiMarco D B \& Jurado R A - The DiMarco-Jurado pleuropericardioplasties: complete closure of the pericardial space with advancement/rotation flaps after adult primary cardiac operations. J Card Surg 1993; 8: 641-9.

12 Berry W R, Klingman R R, Ferraris V A - Pericardial closure without pericardial substitute. Ann Thorac Surg 1993; 55: 1580-1.

13 Schechter F G, Owens R R, Bryant L R - Pleural flap closure of pericardial defects following intrapericardial pneumonectomy. Ann Thorac Surg 1976; 21: 67-9.

14 Kohanna F H, Adams P X, Cunningham Jr. J N, Spencer F C - Use of autologous fascia lata as a pericardial substitute following open-heart surgery. J Thorac Cardiovasc Surg 1977; 74: 14-9.

15 Gallo J I, Pomar J L, Artinano E, Val F, Duran C M Heterologous pericardium for the closure of pericardial defects. Ann Thorac Surg 1978; 26: 149-54.

16 Gabbay S, Bortolotti U, Factor S, Shore D F, Frater R W - Calcification of implanted xenograft pericardium: influence of site and function. J Thorac Cardiovasc Surg 1984; 87: 782-7.

17 Skinner J R, Kim H, Toon R S, Kongtahworn C, Phillips $S \mathrm{~J}$, Zeff $\mathrm{R} \mathrm{H}$ - Inflammatory epicardial reaction to processed bovine pericardium: case report. $J$ Thorac Cardiovasc Surg 1984; 88 (5 Pt 1): 789-91.

18 Mathisen S R, Wu H D, Sauvage L R, Walker M W Prevention of retrosternal adhesions after pericardiotomy. J Thorac Cardiovasc Surg 1986; 92: 92-8.

19 Opie J C, Larrieu A J, Cornell I S - Pericardial substitutes: delayed reexploration and findings. Ann Thorac Surg 1987; 43: 383-5.

20 Heydorn W H, Daniel J S, Wade C E - A new look at pericardial substitutes. J Thorac Cardiovasc Surg 1987; 94 : 291-6.

21 Gabbay S, Guindy A M, Andrews J F, Amato J J, Seaver P, Khan M Y - New outlook on pericardial substitution after open heart operations. Ann Thorac Surg 1989; 48: 803-12.
22 Mazuji M K \& Lett J C - Siliconized dacron as a pericardial patch. Arch Surg 1963; 87: 446-9.

23 Youmans Jr. C R, White J, Derrick J R - The prevention of pleural and pericardial adhesions with silastic. $J$ Thorac Cardiovasc Surg 1968; 55: 383-8.

24 Laks H, Hammond G, Geha A S - Use of silicone rubber as a pericardial substitute to facilitate reoperation in cardiac surgery. J Thorac Cardiovasc Surg 1981; 82: 88-92.

25 Meus P J, Wernly J A, Campbell C D et al. - Long-term evaluation of pericardial substitutes. J Thorac Cardiovasc Surg 1983; 85: 54-8.

26 Amato $\mathrm{J} \mathrm{J}$, Cotroneo J V, Galdieri R J, Alboliras E, Antillon J, Vogel R L - Experience with the polytetrafluoroethylene surgical membrane for pericardial closure in operations for congenital cardiac defects. J Thorac Cardiovasc Surg 1989; 97: $929-34$

27 Lahtinen J, Satta J, Pokela R, Nissinen J, Juvonen T - Pericardial closure with polytetrafluoroethylene surgical membrane or biodegradable polyglycolic acid mesh after coronary artery bypass surgery: a baseline report. Ann Chir Gynaecol 1998; 87:36-9.

28 Liermann A, Lachat M, von Segesser L K, Turina M - Der resorbierbare perikardersatz-eine experimentelle studie. Helv Chir Acta 1991; 58: 515-9.

29 Malm T, Bowald S, Bylock A, Saldeen T, Busch C Regeneration of pericardial tissue on absorbable polymer patches implanted into the pericardial sac: an immunohistochemical, ultrastructural and biochemical study in the sheep. Scand $J$ Thorac Cardiovasc Surg 1992; 26: 15-21.

30 Florez S, Fernandez A L, Herreros J M - Tolerance of a resorbable collagen-elastin membrane as a pericardial substitute in adult cardiac operations. $J$ Thorac Cardiovasc Surg 1999; 117: 185.

31 Mrué F - Substituição do esôfago cervical por prótese biossintética de látex: estudo experimental em cães [Dissertação de Mestrado]. Ribeirão Preto, SP: Faculdade de Medicina de Ribeirão Preto, Universidade de São Paulo, 1996, 109p.

32 Heydorn W H, Ferraris V A, Berry W R - Pericardial substitutes: a survey. Ann Thorac Surg 1988; 46: 567-9.

33 Eng J, Ravichandran P S, Abbott C R, Kay P H, Murday A J, Shreiti I - Reoperation after pericardial closure with bovine pericardium. Ann Thoarc Surg 1989; 48: 813-5.

34 Sussman G L \& Beezhold D H - Allergy to latex rubber. Ann Intern Med 1995; 122: 43-6.

35 Geller M \& Geller P - Considerações sobre a alergia ao látex. JBM 1997; 73: 33-6. 